\title{
Research on the Ranking of University Education based on Grey-TOPSIS-DEA Method
}

\author{
http://dx.doi.org/10.3991/ijet.v10i8.5279 \\ Jianfen Liu, Shengpu Li \\ Pingdingshan University, Pingdingshan, Henan, China
}

\begin{abstract}
The University is the cradle of the higher education. In the college life, the college students continue to accept the new knowledge and continue to grow. Obviously, the level of the university education will be directly related to the growth for the college students. Therefore, it is an important job to evaluate and order the college education quality. In this paper, we combine the Grey theory, TOPSIS with DEA method. And we propose an improved GreyTOPSIS-DEA model. Then, we use the model to evaluate the college education quality. Finally, we get the rankings of the college education. In the last part of this paper, we use the method to evaluate the education quality for different colleges. And we verify the validity of the method.
\end{abstract}

Index Terms-College Education, Evaluation, Ranking.

\section{INTRODUCTION}

In the modern society, the competition is becoming fierce increasingly. Therefore, if we want to get the better development in this society, we must accept the perfect college education. As the place where we accept the high education, the college education quality plays a very important role. Therefore, it is an important job to evaluate and order the college education quality.

M. Ghonji, Z. Khoshnodifar, S.M. Hosseini and S.M. Mazloumzadeh evaluated the education quality of the agricultural higher education institutions. The results showed that the factors that affecting the quality of the agricultural teaching were as follows. (1) lesson plan $(19.52 \%),(2)$ teaching skill $(17.97 \%)$, (3) communication skills $(17.93 \%)$, (4) expertise related to lesson content $(10.59 \%)$, and (5) individual capabilities of members $(9.15 \%)$ respectively[1].Cai Hongmei studied on the quality assurance system of undergraduate teaching in research universities. The author made a systematic research on the quality assurance system of the undergraduate teaching. It is provided a new way and method for the construction of the undergraduate teaching quality assurance system. And it provided the reference for the college teaching quality management personnel [2]. Dong Haiyan researched on the internationalization of the evaluation index and the security system of the undergraduate teaching quality in China. The author expounded the quality principles of the college and the principles of the evaluation index of the teaching quality and the construction of the assurance system. Then, the author analyzed deeply the status, the key and the characteristics of the whole mechanism for the teaching quality evaluation of the domestic and foreign [3]. Lin Xiaoqing improved the ranking method for the static university. Then, he used the dynamic model of the college ranking and used the mathematical example to verify and explain [4]. Xie Yalan researched on indicators and impact of university rankings [5]. From the perspective of the college ranking, Guo Congbin and Sun Qiming compared the world's top universities [6].

The Grey theory is a subject which studied the uncertain information. It was proposed by the Chinese scholar Deng Julong. In recent years, the grey theory continued to develop. It had the wide application on the relation field [7-8] and the prediction field [9-10]. TOPSIS method was based on the close degree between the evaluated objects and the ideal target to rank. The method was to make the relative merits for the existing objects [11-12]. DEA method was a kind of quantitative analysis method. It used the linear programming method, multi input indexes and multi output indexes to do the effective evaluation for the same type units [13-14]. TOPSIS method and DEA method were the main evaluation methods. We can combine and improve the two methods [15-16].

To evaluate and rank the college education quality can promote the university to find out their own shortcomings and corrected. At the same time, it can increase the credibility for the university. In this paper, we combine the Grey theory, TOPSIS with DEA method and propose an improved Grey-TOPSIS-DEA method. Then, we apply the method to the college education quality evaluation. The structure of this paper is as follows. The first part is the introduction. The second part is the basic knowledge. In this paper, we introduce the TOPSIS method and the Grey theory. The third part is the improved Grey-TOPSISDEA method. In this paper, we combine the Grey theory, TOPSIS with DEA method and propose the new evaluated method. The fourth part is the experiment and the last part is the conclusion.

\section{THE BASIC KNOWLEDGE}

\section{A. TOPSIS}

We assume that the scheme set and the attribute set of the multi attribute decision problem are $A=\left\{A_{1}, A_{2}, \mathrm{~L}, A_{m}\right\}$ and $F=\left\{f_{1}, f_{2}, \mathrm{~L}, f_{n}\right\}$. The decision matrix is $B=\left\{b_{i j}\right\}_{m \times n}$. The scheme $A_{i}$ is written as $A_{i}=\left(b_{i 1}, b_{i 2}, \mathrm{~L}, b_{i n}\right), i=1,2, \mathrm{~L}, m, b_{i j} \geq 0$. The weight vector of the attribute is $W=\left(\omega_{1}, \omega_{2}, \mathrm{~L}, \omega\right)^{T}$ and $\sum_{j=1}^{n} \omega_{j}=1, \omega_{j} \geq 0, j=1,2, \mathrm{~L}, n$. The step of the traditional TOPSIS method is as follows. 
Firstly, we use the vector norm method to construct the decision matrix $C=\left(c_{i j}\right)_{m \times n}$. For the benefit attribute,

$$
c_{i j}=\frac{b_{i j}}{\sqrt{\sum_{i=1}^{m} b_{i j}^{2}}}
$$

For the cost attribute,

$$
c_{i j}=\frac{1 / b_{i j}}{\sqrt{\sum_{i=1}^{m}\left(1 / b_{i j}\right)^{2}}}
$$

Where, $i=1,2, \mathrm{~L}, m, j=1,2, \mathrm{~L}, n$.

Secondly, we weight the normalized decision matrix and get the weighted normalized matrix $Z=\left(z_{i j}\right)_{m \times n}$. Where $z_{i j}=\omega_{i j} c_{i j}, i=1,2, \mathrm{~L}, m, j=1,2, \mathrm{~L}, n$.

The third step is as follows. After we get the normalized matrix, we need to determine the positive ideal solution $A^{+}$and the negative ideal solution $A^{-}$. Then defining two artificial scheme.

The positive ideal scheme and the negative ideal scheme are as follows.

$$
\begin{aligned}
& A^{+}=\left(z_{1}^{+}, z_{2}^{+}, \mathrm{L}, z_{n}^{+}\right) \\
& A^{-}=\left(z_{1}^{-}, z_{2}^{-}, \mathrm{L}, z_{n}^{-}\right)
\end{aligned}
$$

For the benefit attribute,

$z_{j}^{+}=\max _{i} z_{i j}, z_{j}^{-}=\min _{i} z_{i j}$

For the cost attribute,

$$
z_{j}^{+}=\min _{i} z_{i j}, z_{j}^{-}=\max _{i} z_{i j}
$$

The fourth step is to calculate the distance from each scheme to the positive ideal solution $d_{i}^{+}$and the negative ideal solution $d_{i}^{-}$.

$$
\begin{aligned}
& d_{i}^{+}=\left\|\mathrm{z}_{i}-A^{+}\right\|=\sqrt{\sum_{j=1}^{n}\left(z_{i j}-z_{j}^{+}\right)^{2}} \\
& d_{i}^{-}=\left\|\mathrm{z}_{i}-A^{-}\right\|=\sqrt{\sum_{j=1}^{n}\left(z_{i j}-z_{j}^{-}\right)^{2}}
\end{aligned}
$$

Where $i=1,2, \mathrm{~L}, m, j=1,2, \mathrm{~L}, n, z_{i}=\left(z_{i 1}, z_{i 2}, \mathrm{~L}, z_{i n}\right)$.

The fifth step is to calculate the relative closeness $C_{i}^{+}$ between each scheme and the positive ideal solution.

Where, $i=1,2$, L , $m$.

$$
C_{i}^{+}=\frac{d_{i}^{-}}{d_{i}^{+}+d_{i}^{-}}
$$

It can be seen if $z_{i}=A^{+}, C_{i}^{+}=1$. And if $z_{i}=A^{-}$, $C_{i}^{+}=0.0 \leq C_{i}^{+} \leq 1$. When $C_{i}^{+} \rightarrow 1$ the scheme $A_{i} \rightarrow A^{+}$.

Lastly, we rank $C_{i}^{+}$according to the descending order.

\section{B. Grey relation}

The grey system theory provides the index quantitative measurements for the project selection decision. We do the grey correlation degree analysis according to the same factors for each scheme. Then we get the numerical relationship of the same factor for each scheme. The step of the grey correlation method is as follows.

Firstly, we establish the index matrix $A=\left[x_{i j}\right]_{m \times n} \cdot m$ is the number of the preferred items. $n$ is the number of the indexes. The weight of each index is $W=\left(w_{1}, w_{2}, \mathrm{~W}_{3}, \mathrm{~L}, w_{n}\right)$.

Secondly, we give the weight on the basis of the index matrix $A$. And we construct the weighted matrix $B: B=A W$.

Thirdly, according to the weighted index matrix, we can get the positive ideal scheme $a^{+}$and the negative ideal scheme $a^{-}$.

The fourth step is to calculate the correlation between each scheme and the positive scheme and the negative scheme.

$$
L_{i j}=\frac{\min _{i} \min _{j}\left|a_{0 j}-a_{i j}\right|+\theta\left|a_{0 j}-a_{i j}\right|}{\left|a_{0 j}-a_{i j}\right|+\theta \max _{i} \max _{j}\left|a_{0 j}-a_{i j}\right|}
$$

For $a_{0}, L_{i j}$ is the correlation coefficient at $j$. The value range for the correlation coefficient is $[0,1]$. If expresses that the similar degree of the two factors which are compared at one point. Where, $\theta$ is the distinguish coefficient. It expresses the distortion that is caused by weakening the biggest absolute difference value. The value range for $\theta$ is $[0,1]$. According to the experience value, $\theta=0.5 . i=1,2, \mathrm{~L}, j=1,2, \mathrm{~L}, n$. The overall correlation of the scheme is the average of all kinds of the correlation coefficient. That is,

$$
R_{i}=\frac{1}{n} \sum_{j=1}^{n} L_{i j}
$$

The fifth step is to calculate the relative close degree between each scheme and the ideal scheme.

$$
r_{i}=\frac{R_{i}^{+}}{R_{i}^{+}+R_{i}^{-}}
$$

The sixth step is as follows. The optimal scheme is the scheme that the relative degree is bigger. Then we order the alternative scheme according to the size of the close degree.

\section{THE IMPROVED GREY-TOPSIS-DEA}

We assume that there are $n$ indexes $x_{1}, x_{2}, \mathrm{~L}, x_{n}$. Correspondingly, it exists the optimal ideal index set $x^{*}=\left\{x_{1}^{*}, x_{2}^{*}, \mathrm{~L}, x_{n}^{*}\right\}$ and the worst index set $\tilde{x}^{\sim}=\left\{\tilde{x}_{1}, \tilde{x}_{2}, \mathrm{~L}, \tilde{x}_{n}\right\}$. The weight of each index is $\omega_{1}, \omega_{2}, \mathrm{~L}, \omega_{n}$. Now, there are $m$ assessment scheme $y^{1}, y^{2}, \mathrm{~L}, y^{m}$. The index of each scheme is $\left\{x_{1}^{1}, x_{2}^{1}, \mathrm{~L}, x_{n}^{1}\right\},\left\{x_{1}^{2}, x_{2}^{2}, \mathrm{~L}, x_{n}^{2}\right\}, \mathrm{L},\left\{x_{1}^{m}, x_{2}^{m}, \mathrm{~L}, x_{n}^{m}\right\}$. Where, $x_{k}^{i}$ is the maximum index which is processed by the dimensionless and $x_{k}^{i} \in[0,1] . \alpha_{k}^{i}$ is the correlation coefficient of the $k$ index for the $i$ scheme and the $k$ optimal ideal index. $\beta_{k}^{i}$ is the correlation coefficient of the $k$ index for the $i$ scheme and the $k$ worst ideal index. 


$$
\begin{aligned}
& \alpha_{k}^{i}=\frac{\min _{i} \min _{k}\left|x_{k}^{*}-x_{k}^{i}\right|+\rho \cdot \max _{i} \max _{k}\left|x_{k}^{*}-x_{k}^{i}\right|}{\left|x_{k}^{*}-x_{k}^{i}\right|+\rho \cdot \max _{i} \max _{k}\left|x_{k}^{*}-x_{k}^{i}\right|} \\
& \beta_{k}^{i}=\frac{\min _{i} \min _{k}\left|x_{k}^{\sim}-x_{k}\right|+\rho \cdot \max _{i} \max _{k}\left|x_{k}^{\sim}-x_{k}^{i}\right|}{\left|x_{k}^{\sim}-x_{k}^{i}\right|+\rho \cdot \max _{i} \max _{k}\left|x_{k}^{\sim}-x_{k}^{i}\right|}
\end{aligned}
$$

We assume that $S_{+}^{i}$ is the distance from the $i$ scheme which is processed by the standardized to the optimal ideal index set. $S_{-}^{i}$ is the distance from the $i$ scheme which is processed by the standardized to the worst ideal index set .

$$
\begin{aligned}
& S_{+}^{i}=\frac{\overline{S_{+}^{i}}-\overline{S_{+}^{*}}}{\overline{S_{+}^{\sim}-\overline{S_{+}^{*}}}} \\
& S_{-}^{i}=\frac{\overline{S_{-}^{i}}-\overline{S_{-}^{\sim}}}{\overline{S_{+}^{*}-\overline{S_{-}^{\sim}}}}
\end{aligned}
$$

Where, $\overline{S_{+}^{i}}$ is the distance from the $i$ scheme which is not processed by the standardized to the optimal ideal index set and $\overline{S_{+}^{i}}=\sum_{k=1}^{n}\left(\omega_{k} \cdot \beta_{k}^{i}\right) . \overline{S_{-}^{i}}$ is the distance from the $i$ scheme which is not processed by the standardized to the worst ideal index set and $\overline{S_{-}^{i}}=\sum_{k=1}^{n}\left(\omega_{k} \cdot \alpha_{k}^{i}\right) \cdot \overline{S_{+}^{*}}$ is the distance from the optimal ideal index set which is not processed by the standardized to the optimal ideal index set and $\overline{S_{+}^{*}}=\sum_{k=1}^{n}\left(\omega_{k} \cdot \beta_{k}^{*}\right) \cdot \overline{S_{-}^{i}}$ is the distance from the optimal ideal index set which is not processed by the standardized to the worst ideal index set and $\overline{S_{-}^{*}}=\sum_{k=1}^{n}\left(\omega_{k} \cdot \alpha_{k}^{*}\right) \cdot \overline{S_{+}^{\sim}}$ is the distance from the worst ideal index set which is not processed by the standardized to the optimal ideal index set and $\overline{S_{+}^{\sim}}=\sum_{k=1}^{n}\left(\omega_{k} \cdot \beta_{k}^{\sim}\right) . \overline{S_{-}^{\sim}}$ is the distance from the worst ideal index set which is not processed by the standardized to the worst ideal index set and $\overline{S_{-}^{\sim}}=\sum_{k=1}^{n}\left(\omega_{k} \cdot \alpha_{k}^{\sim}\right)$.

$C^{i}$ is the relative closes degree of the $i$ scheme for the ideal index set.

$$
C^{i}=\frac{S_{-}^{i}}{S_{-}^{i}-S_{+}^{i}}
$$

Where, $C^{i}$ is the evaluation value that the method gives.

Then, we use DEA method to adjust the result for $C^{i}$.

$\delta_{\beta}$ is the output efficiency and the $\delta_{\alpha}$ is the input efficiency. We notice that when $\delta_{\beta}$ is close to zero or $\delta_{\alpha}$ is greater than 1 , the adjusted degree becomes bigger. When $\delta_{\beta}$ is close to 1 or $\delta_{\alpha}$ is close to 1 , the adjusted degree becomes smaller. The adjusted formula for DEA method is as follows.

$$
C_{i}=C^{i}+C^{\prime}
$$

Where,

$$
C^{\prime}=\frac{1}{2} C^{i}\left(1-\delta_{\alpha} \delta_{\beta}\right)
$$

$C_{i}$ is the evaluation value which is adjusted by the DEA method.

The flow chart is shown in the following figure.

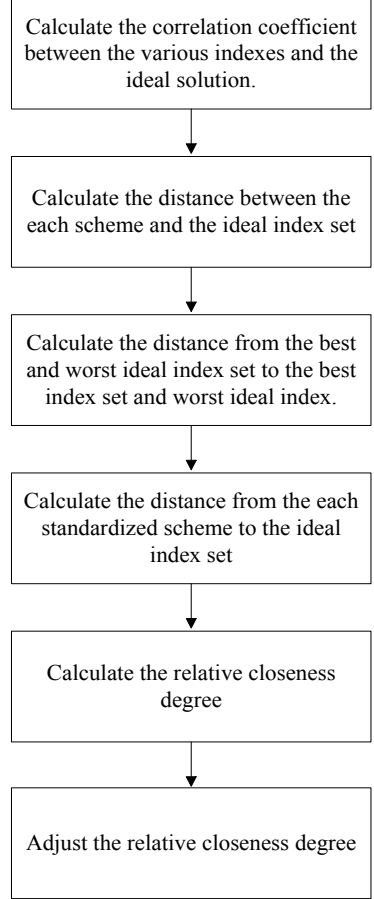

Figure 1. The flow chart of the improved Grey-TOPSIS-DEA method

\section{EXPERIMENT}

In this paper, we rank the universities according to the teaching quality. Firstly, we establish the college teaching quality evaluation system. The college teaching quality evaluation system is as follows.

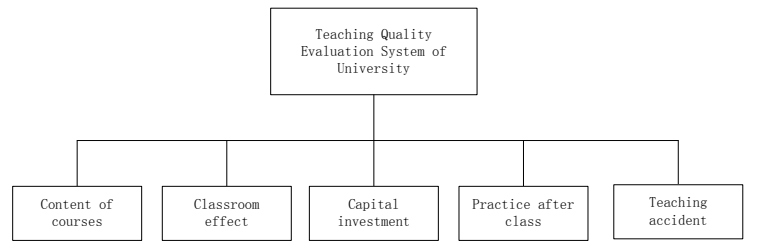

Figure 2. college teaching quality evaluation system

Firstly, we get the weight of each index $W=(0.21,0.34,0.14,0.23,0.08)$. Then we rank the teaching quality the six universities $\left\{A_{1}, A_{2}, A_{3}, A_{4}, A_{5}, A_{6}\right\}$. The decision matrix is as follows.

$$
C=\left[\begin{array}{lllll}
8 & 6 & 7 & 9 & 7 \\
6 & 6 & 9 & 7 & 8 \\
7 & 8 & 7 & 9 & 8 \\
7 & 9 & 8 & 6 & 7 \\
9 & 6 & 7 & 9 & 8 \\
6 & 7 & 9 & 8 & 6
\end{array}\right]
$$

We can get the evaluation result. 
TABLE I.

THE RANK FOR THE UNIVERSITY WHICH IS NOT ADJUSTED

\begin{tabular}{|c|c|c|}
\hline university & $C^{i}$ & rank \\
\hline$A_{1}$ & 0.487 & 5 \\
\hline$A_{2}$ & 0.529 & 4 \\
\hline$A_{3}$ & 0.712 & 1 \\
\hline$A_{4}$ & 0.534 & 3 \\
\hline$A_{5}$ & 0.450 & 6 \\
\hline$A_{6}$ & 0.613 & 2 \\
\hline
\end{tabular}

Then, we use the DEA method to adjust. The results are as follows.

TABLE II.

THE RANK FOR THE UNIVERSITY WHICH IS ADJUSTED

\begin{tabular}{|c|c|c|c|}
\hline university & Before adjustment & After adjustment & rank \\
\hline$A_{1}$ & 0.487 & 0.465 & 5 \\
\hline$A_{2}$ & 0.529 & 0.544 & 3 \\
\hline$A_{3}$ & 0.712 & 0.723 & 1 \\
\hline$A_{4}$ & 0.534 & 0.528 & 4 \\
\hline$A_{5}$ & 0.450 & 0.452 & 6 \\
\hline$A_{6}$ & 0.613 & 0.632 & 2 \\
\hline
\end{tabular}

\section{CONCLUSIONS}

The college education is very important for a person's growth. The college is not only the place where we study, but also the temple of the knowledge. To evaluate the college education and get the rankings is a very important job. In this paper, we combine the Grey theory, TOPSIS with the DEA method and propose an improved GreyTOPSIS-DEA method. Then we use the model to evaluate the college education quality and get the rankings of the college education. The main job of this paper is as follows. Firstly, we introduce the background of the university education evaluation. Secondly, we introduce the Grey theory and TOPSIS method. Thirdly, we propose an improved Grey-TOPSIS-DEA model. And we apply the method to the quality evaluation of the college education. The experiment in this paper shows the effectiveness.

\section{REFERENCES}

[1] M. Ghonji, Z. Khoshnodifar, S.M. Hosseini, S.M. Mazloumzadeh. Analysis of the some effective teaching quality factors within faculty members of agricultural and natural resources colleges in Tehran University [J].Journal of the Saudi Society of Agricultural Sciences, 2015, 14(2): 109-115. http://dx.doi.org/10.1016/ j.jssas.2013.04.003

[2] Cai Hongmei. Study on the quality assurance system of undergraduate teaching in research universities [D]. Huazhong University of Science and Technology, management science and engineering, 2015.

[3] Dong Haiyan. The research on the internationalization of the evaluation index and the security system of the undergraduate teaching quality in China [D]. Nanjing University of Science and Technology, Higher education, 2004.

[4] Lin Xiaoqing. Improving the methods of university ranking [J]. Educational Research, 2009, 11: 27-35.

[5] Xie Yalan. Research on Indicators and Impact of University Rankings [D]. Shanghai Jiao Tong University, University Evaluation, 2010.
[6] Guo Congbin, Sun Qiming. A Comparative Analysis of Chinese Universities and the World-class Universities - From the Perspective of University Rankings [J]. Educational Research, 2015, 2: 147-157.

[7] Xiaoliang Jia, Haizhong An. Finding the Interdependence Among Various Crude Oil Prices: A Grey Relation Network Analysis [J].Energy Procedia,2015,75: 2563-2568. http://dx.doi.org/10.10 16/j.egypro.2015.07.296

[8] Qi Wang, Chong Wu, Yang Sun. Evaluating corporate social responsibility of airlines using entropy weight and grey relation analysis [J].Journal of Air Transport Management, 2015,42: 5562. http://dx.doi.org/10.1016/j.jairtraman.2014.08.003

[9] Che-Jung Chang, Der-Chiang Li, Yi-Hsiang Huang, Chien-Chih Chen.A novel gray forecasting model based on the box plot for small manufacturing data sets [J]. Applied Mathematics and Computation, 2015, 265: 400-408. http://dx.doi.org/10.1016/ j.amc.2015.05.006

[10] Rotchana Intharathirat, P. Abdul Salam, S. Kumar, Akarapong Untong. Forecasting of municipal solid waste quantity in a developing country using multivariate grey models $[\mathrm{J}]$. Waste Management, 2015, 39: 3-14. http://dx.doi.org/10.1016/j.wasman. 2015.01.026

[11] Zhi Pei. A note on the TOPSIS method in MADM problems with linguistic evaluations [J].Applied Soft Computing, 2015, 36: 2435. http://dx.doi.org/10.1016/j.asoc.2015.06.042

[12] Peter Wanke, Carlos Pestana Barros, Zhongfei Chen. An analysis of Asian airlines efficiency with two-stage TOPSIS and MCMC generalized linear mixed models [J]. International Journal of Production Economics, 2015, 169:110-126 http://dx.doi.org/10.1016/ j.ijpe.2015.07.028

[13] Sungmook Lim, Joe Zhu. A note on two-stage network DEA model: Frontier projection and duality. European Journal of Operational Research, 2016, 248: 342-346. http://dx.doi.org/10.1016/ j.ejor.2015.06.050

[14] He-Boong Kwon, Jooh Lee. Two-stage production modeling of large U.S. banks: A DEA-neural network approach [J].Expert Systems with Applications, 2015, 42(19): 6758-6766. http://dx.doi.org/10.1016/j.eswa.2015.04.062

[15] Aydın Çelen, Neşe Yalçın. Performance assessment of Turkish electricity distribution utilities: An application of combined FAHP/TOPSIS/DEA methodology to incorporate quality of service $[\mathrm{J}]$. Utilities Policy, 2012, 23: 59-71. http://dx.doi.org/10.1016/j.jup.2012.05.003

[16] M. Amiri, M. Zandieh, B. Vahdani, R. Soltani, V. Roshanaei. An integrated eigenvector-DEA-TOPSIS methodology for portfolio risk evaluation in the FOREX spot market [J]. Expert Systems with Applications, 2010, 37: 509-516. http://dx.doi.org/10.1016/ j.eswa.2009.05.041

\section{AUTHORS}

Jianfen Liu is an Associate Professor at College of Computer Science and Technology, Pingdingshan University, Pingdingshan, Henan, 467000, China. She received the B.Sc., M.Sc., degrees from Henan Normal University and Wuhan University of Science and Technology, Xinxiang and Wuhan, China, in 1995 and 2003 respectively. Her research interests include: Network Information Processing, and Intelligent Algorithm. (email: 2094991521@qq.com)

Shengpu Li is a Lecturer at College of Computer Science and Technology, Pingdingshan University, Pingdingshan, Henan, 467000, China. He received the B.Sc., M.Sc., degrees from Harbin University of Commerce and Xidian University, Harbin and Xian, China, in 2005 and 2010 respectively. His research interests include: The Internet of Things Technology and Application. (email: Lsp1519@163.com)

Submitted 07 November 2015. Published as resubmitted by the authors 05 December 2015. 